\title{
A new radio-frequency acoustic method for remote study of liquids
}

\author{
Alexander V. Kramarenko,*, Andrey V. Kramarenko ${ }^{\mathrm{b}}$ \\ ${ }^{a}$ TREDEX Company Ltd., PO box 11515, Kharkiv, Ukraine 61001 \\ ${ }^{b}$ General and Inorganic Chemistry Department, National Technical University "KhPI", 2 Kyrpychova str, Kharkiv, Ukraine 61002
}

\begin{abstract}
In the present work a new method of study of liquids has been proposed. It is based on phenomenon of radio frequency anisotropy of electrolyte solution discovered by us. It arises because of mechanical or acoustic excitation of the solution. We were observing the phenomenon during the development process of RF polarimetric contactless cardiograhpy. The electric field vector of transmitted 433.82 MHz signal becomes rotated after its transition through the pericardial region. That rotation depends on change of blood acceleration when passing through the chambers of the heart and large vessels. It has also been revealed that rotation occurs after $\mathrm{RF}$ wave passage through the physiological saline $(0.9 \% \mathrm{NaCl})$ subjected to any mechanical excitation inside it like a jet appearing or soundwave passing. No significant difference was detected experimentally between $\mathrm{NaCl}$ and $\mathrm{KCl}$ solutions behaviour. It means that the mechanism of hydrodynamic separation of ions is apparently not suitable to explain the phenomenon. The response we have registered most likely resembles the magnetization process of spin glasses. From the nature of the response observed we have concluded that a fundamentally new physical effect is discovered. It may provide wide opportunities for remote measurement of the electrolyte solutions parameters using polarized radio-frequency signals.
\end{abstract}

Keywords: contactless cardiography, polarization vector, polarimetry, aqueous electrolyte solution, relaxation time, spin configuration

\section{Introduction}

Back in 1845, Michael Faraday predicted the phenomenon of electric field vector rotation of linearly polarized light in an external magnetic field applied. And when John Kerr discovered optical material refractive index change proportional to the square of the external electric field strength, widespread use of this just discovered electro-optical phenomenon began. The usage of certain substance indirectly changing the polarization of electromagnetic radiation passing through it was a necessary condition for Kerr, Pockels (and other) cells to work. In terms of radiophysics one may represent it like parametric influence to the system consisting of transmitter, wave propagation in medium and receiver. Hence the hypothesis presuming existence of Faraday phenomenon at radio frequencies looks plausible enough.

In present work we propose a completely new method of conductive liquids study based on physical phenomenon that has never been observed earlier, as far as we know. The phenomenon is the radio-frequency anisotropy of electrolyte solution that is excited mechanically or acoustically. We suppose the method can be used as in fundamental physical experiment, as in a variety of areas of industry, biology and medicine, also as in oceanography and geology.

\footnotetext{
* Principal corresponding author

Email addresses: tredexcompany37@gmail.com (Alexander V. Kramarenko), kramarenko@kpi.kharkov . ua (Andrey V. Kramarenko)
}

The difference of electric potential between two points of electrolyte solution, which is moving with variable acceleration, was discovered earlier [1] (english abstract [2], remastered english version [3]). However, the polarization direction rotation was not a matter this paper dealt with.

The effect described in present work have unexpectedly been discovered while testing the device we were designing. It is a new type of fully contactless cardiograhpy which is important for assessment of human health status when the application of ECG electrodes is impossible or inconvenient.

A mechanism of rotation of so-called medium polarization vector of scattering (PVS [4] is a real part of Jones vector in our case) was assumed to be obvious at the beginning of polarimetric cardiography designing. It is known that the miocard and large vessels connected to it are deforming and rotating during the systole. It has long been established reliably by dynamic studies of cardiac activity [5]. Indeed, the radio wave passing through the pericardial region will change its polarization direction because of the heterogeneous tissues shifting and rotating. It means that expected rotation of polarization direction might be synchronized with an "external" heart ballistics.

Figure 1 shows a signal pathway block diagram. RF signal of transmitter 1 excites the transmitting antenna 2. A linearly polarized wave passes through the object 3 (some abstract medium is shown here) to be received then by two orthogonal oriented antennas 5. Input stages 6 (X channel) and 7 (Y channel) amplify the corresponding signals that pass then to amplitude logarithmic detectors 8 and 9. Differential amplifier 10 provides an output signal which is proportional to the differ- 


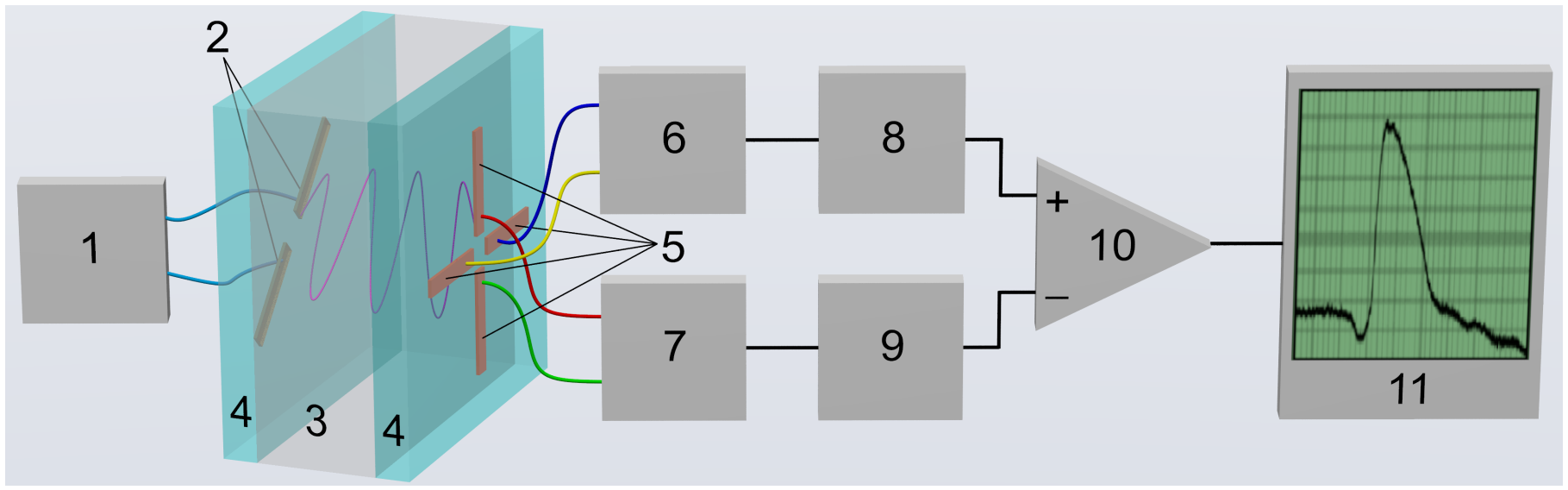

Figure 1: A simplified block diagram of the device for recording of RF wave polarization direction deviation.

1 - a continuous wave transmitter $433.82 \mathrm{MHz},-12 \mathrm{dBm} ; 2$ - transmitting antenna; 3 - an object studied; 4 - a dielectric material; 5 - orthogonal orientation quadrature antennas; 6 - channel X RF amplifier; 7 - channel Y RF amplifier; 8 - X channel amplitude logarithmic detector; 9 - Y channel amplitude logarithmic detector; 10 - instrumentation differential amplifier; 11 - registrating device (X axis is time, Y axis is deviation). The antennas are shown as Hertz's half-wave dipoles, it is clear that their real configuration will be different. Transmitting antenna is deviated at angle of $45^{\circ}$ relative to both receiving ones.

ence in amplitudes of low-frequency signals. A standard (for ECG recording) bandwidth having the low-frequency cutoff at $0.3 \mathrm{~Hz}(-3 \mathrm{~dB})$ is applied. In order to observe the dynamics of the process, an oscilloscope 11 records the time dependence of the value of PVS projection to the plane normal to wavevector.

So, the multistatic radar, emitting a continuous wave throughpassing the pericardial region, has been implemented.

Thus, we have successfully detected polarization change of UHF band wave. The cardiogram expected having a minimum of artifacts and interference was recorded by the contactless method [6]. Figure 2 shows a polarimetric cardiogram recorded synchronously with a standard ECG.

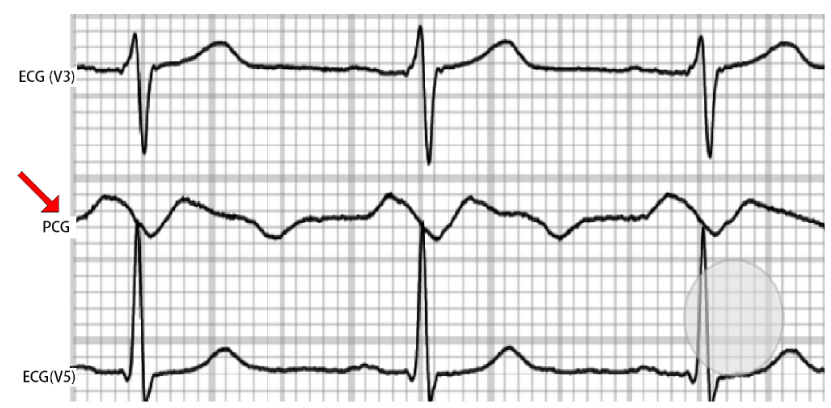

Figure 2: The polametric cardiogram (PCG) recorded synchronously together with a standard ECG (ECG (V3), ECG (V5))

But the polarimetric cardiogram (PCG) curves are in contrary with initial hypothesis. They are more prolonged so that they starting earlier than ECG and finishing later than it which is defied explanation. PCG signal is in a good accordance with time stamps of intracardiac (NB!) hemodynamics. It has been turned out than PVS vector rotation is associated rather with "internal" cardiac ballistics than with "external" one. In other words, such a rotation caused by moving of intracardiac and intravascular blood (inside the heart and large vessels connected to it).

We hypothesized that radio wave polarization direction after its passing through the pericardial region depends on electric properties of the medium itself i.e. on the properties of the blood this radio wave passing through.

Also we assumed that the blood which is immobile (or moving steady) may rotate a radio wave polarization direction by some (zero or non-zero) constant angle that should not change in time. The variations in rotation angle could only be come with blood acceleration or deceleration, i.e. a non stationary blood motion. Figure 2 shows that the beginning of the PVS deviation coincides with the peak of $\mathrm{P}$ wave on the ECG. It corresponds to the beginning of an intracardiac discharge of blood into the ventricles when myocard just starting to contract, but blood is still almost motionless. This coincidence is a good confirmation of our assumption. Moreover, a non-zero PCG signal was recorded even after the cardiac contraction has finished when only the reverse blood flow occurs in aorta and pulmonary artery, which closes the heart valves with a sudden bloodstream slowdown.

Thus, we have the right to assume that the PVS rotation is caused not only by heart displacement and rotation but rather by variation of properties of moving blood (which is a solution of electrolytes).

At the first consideration we assumed the mechanism of anisotropy occurence as next. When the liquid moves with variable acceleration, the hydrodynamic forces drive cations and anions differently. It may occur due to non equal masses of ions, theirs different radii and hydration numbers. Hence the displacement of negative charges relative to positive ones might arise. Such a displacement, in turn, should cause the occurence of parallel oriented statistic dipole structures in solution. Then the polarization direction of radio signal passed through it will rotate because of the ortogonally polarized waves have different speeds in anisotropic medium.

But our further experiments and calculations showed the 


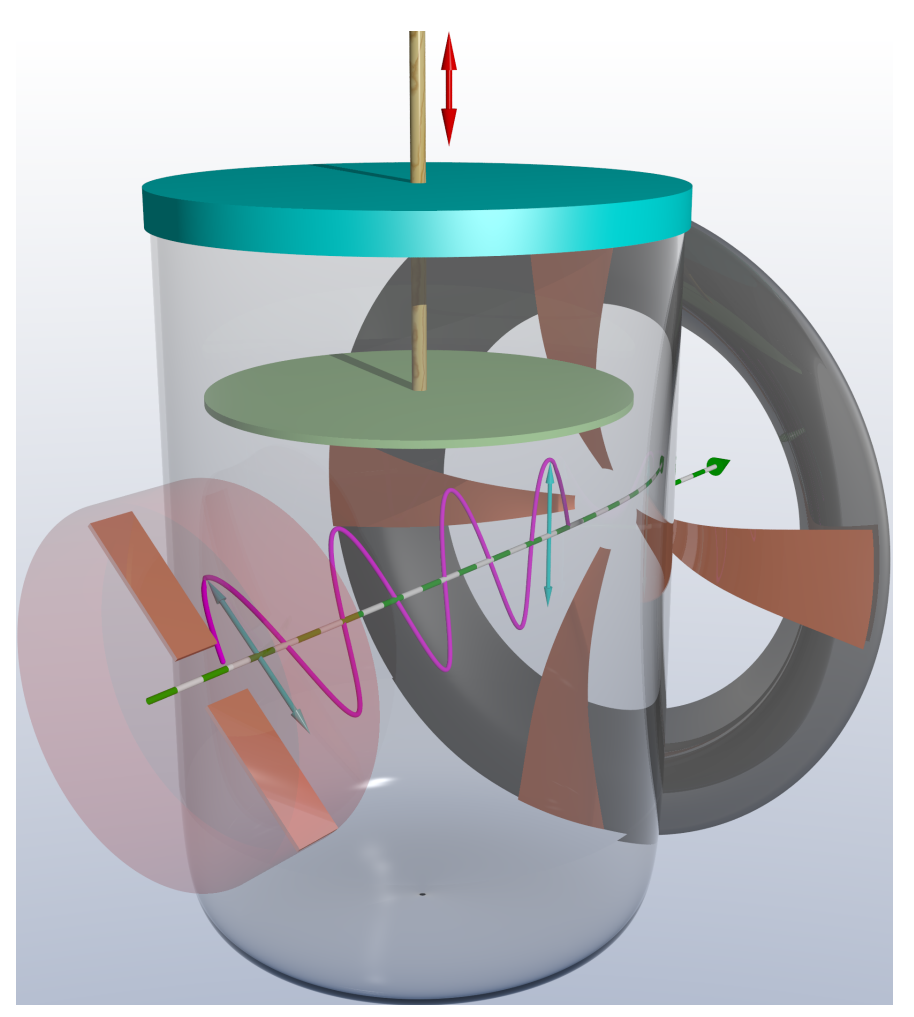

Figure 3: The experimental setup scheme. A shortened transmitting antenna (mounted in a radar absorbing case) is on the left while a receiving quadrature slot antenna is on the right. The relative orientation of the antennas polarization is $45^{\circ}$

failure of such an approach.

\section{Experimental}

In order to test experimentally our hypothesis about the dependence of PVS rotation on the blood movement as a conductive medium we assembled the setup which figure 3 shows. Between the transmitting antenna and receiving one a container is placed filled with an electrolyte solution under the lid itself, without air residues. This was done so that the solution does not sway when the disk moves, and thus does not change its shape as a whole, thereby distorting the scattering field and affecting the measurement results. Inside the container there is a thin polystyrene disk mounted on a long dielectric pusher. The disk was moving in the solution because of an external force acting through the pusher. An experiment has been carried out at ambient temperature of $+20^{\circ} \mathrm{C}$.

After the recording equipment has been turned on the disk was displacing in axial direction by the pusher because of the weight dropping. The falling of the weight was provided either by a soft push through the shock-absorbing pad or with a sharp blow. The absence of any responses has been checked previously when the disk was moving inside the empty container and when it was filled with distilled water. In the first case, there was no signal at all. In the second a barely noticeable signal was recorded at the level of noises. Response signal disappeared completely when replacing the distilled water with a

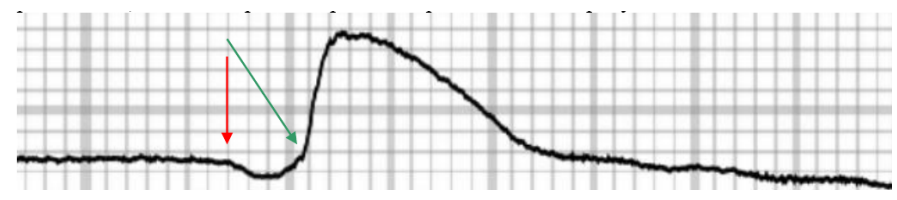

Figure 4: Deviation of the polarization direction during a soft push of the disk (the beginning and end of the external force are marked by arrows). In the experiment with tinting the solution, it was seen that the jets flowing around the disk move in the opposite direction at a higher speed than the disk itself. The grid has a minimum horizontal division of $40 \mathrm{~ms}$ and approximately $4^{\circ}$ vertical rotation of the direction (the vertical scale, unfortunately, is non-linear)

bidistilled one. One more experiment has also been carried out with distilled water in which citric acid was dissoling slowly. The response was increasing while the acid was dissolving. In this experiment the same RF equipment was used as for cardiograms recording.

When the container was filled with isotonic $\mathrm{NaCl}$ solution, any displacement of the disk always caused the appearance of a high-amplitude signal at the detector output. It should be noted that this experiment is easy to implement and is highly repeatable. Despite this, the effect has gone unnoticed since the invention of radio communications. Figures 4 and 5 show the waveforms of the process.

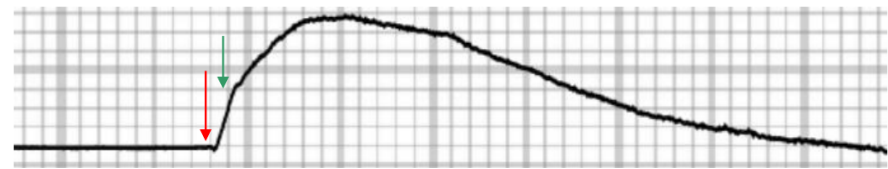

Figure 5: The same with a sharp hit on the pusher (the jets flowing differs from the previous observation)

The amplitude of the output signal increased with increasing the pusher acceleration. An experiment with more concentrated solutions also demonstrated that the response also increased with $\mathrm{NaCl}$ concentration growth ceteris paribus. With further increase of concentration a response passed through a maximum of intensity, then decreased as the concentration approached the saturation, and almost ceased to be observed for a saturated solution. Here we can make the assumption that the described effect is recorded by our equipment only for sufficiently concentrated but unsaturated solutions. No experiments with radio frequency variation were carried out. We have chosen the frequency so that the half-wavelength approximates the linear dimensions of the heart and large vessels.

We did not find detectable differences in the response for $\mathrm{NaCl}$ and $\mathrm{KCl}$ solutions having approximately the same molar concentrations.

In order to find the probable acoustic phenomenon we have also recorded the response from the solution excited with a hydrophone immersed into the solution being in container. A hydrophone was driven using MOSFET switch mode output stage. Figures 6 and 7 show the corresponding waveforms recorded from the operational amplifier output.

After the equipment reconstrution in order to expand the 


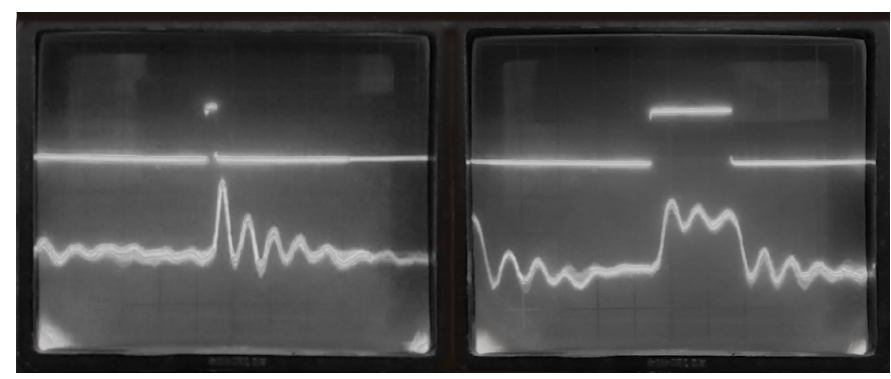

Figure 6: Left waveforms show a system response on the repetitive acoustic pulse $1 \mathrm{~ms}$ long. Waveforms on the right show both the pulse duration and repetition frequency increased. The damped oscillatory process is clearly visible, possibly due to an echo in the container. Upper beam shows a hydrophone input signal while the lower one is the PVS rotation. An electric power of the impulse applied to the hydrophone is equal to $1 \mathrm{~mJ}$. The polarization direction of the wave is deviated from the soundwave propagation vector by $45^{\circ}$. We were able to observe the effect confidently down to pulse energies of $1 \mu \mathrm{J}$

bandwidth to zero frequency we have revealed a completely unexpected behavior of the system studied. A reaction of electrolyte solution because of acoustic wave front passage was very prolonged contrary to our expectations. Thus, an undoubted long-term (in comparison with typical solvent relaxation times) "memory" was detected for the system. In addition a slow PVS rotation continued right after its rapid deviation at the moment of the acoustic wave passage so that the degree of anisotropy of the entire volume was increasing (see fig. 7) within tens of seconds after the pulse. The PVS rotation angle value passed through a maximum and returned then to its starting values (before the pulse) after 30 seconds or more.

In order to exclude the influence of RF energy "pumping" into the object studied we have attempted to interfere to the process with another transmitter which was more powerful $(+20 \mathrm{~dB})$ than the used one and had the same frequency (but different polarization direction). It was turning on for a very short time but had no effect on the process of post-impulse PVS rotation (see fig. 7). It is obvious that we can neglect the "observer effect", i.e. there is no changes in the structure of the object observed because of the radio signal passing through it.

Our experiments cannot yet provide a quantitative estimation of the phenomenon registered that is outwardly similar to the effects discovered by Kerr and Pokelson. It is not mentioned in the main physical reference books (for example, [7]). However, we have experimentally established now that the unsteady motion of electrolyte solution affects the polarization direction of the RF wave passing through it.

Summarizing the observations we have carried out it should be necessary to highlight the next facts:

1. Acoustic or mechanical excitation of pure water does not cause a PVS rotation we can detect with the equipment available. Supporting the constant level of acoustic excitation we have revealed that the PVS rotation angle increases with increasing concentration of electrolyte in water. The response decreased when approaching the saturation of $\mathrm{NaCl}$ solution, and for the saturated solution it practically ceased to be observed using our equipment.
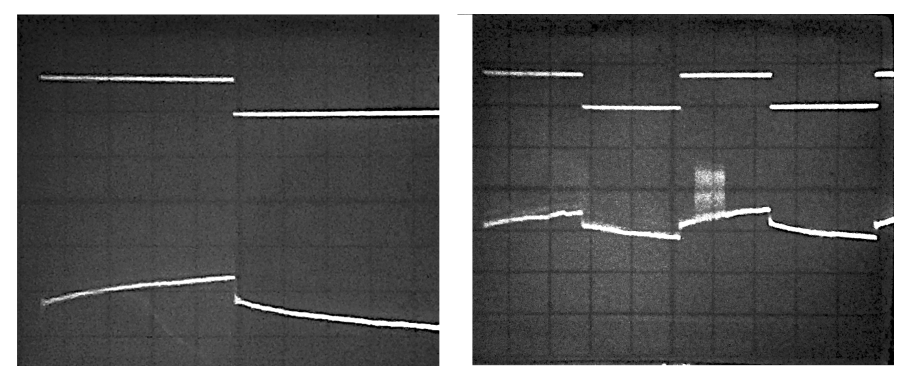

Figure 7: A system response on the Heaviside function signal (upper beam) is shown on the left. Recording duration is $2.5 \mathrm{~s}$. The equipment bandwidth starts at zero frequency. It is easy to observe that after a rapid, momentary PVS rotation because of the acoustic wave propagation, the further post-impulse rotation continues in the same direction. On the right is a $5 \mathrm{~s}$ fragment of the record that shows an unsuccessful attempt to interfere to post-impulse PVS rotation process by turning on (briefly) an additional more powerful transmitter. Interference is visible on the lower beam of the oscilloscope

2. An experiment with $\mathrm{KCl}$ solution having a molar concentration of $0.157 \mathrm{~mol} / \mathrm{l}$, which is close to that for an isotonic $\mathrm{NaCl}$ solution, did not reveal an experimentally detectable difference in response between them.

3. The magnitude of the resulting RF anisotropy depends on the energy of the front of the acoustic wave passing through the electrolyte solution and does not depend on the power of the RF transmitter.

4. When the wave front passes through, anisotropy arises almost inertialessly, i.e. the times observed were less than $50 \mu \mathrm{s}$ at the existing installation (and they are most likely much less than this value).

5. Anisotropy persists for at least tens of seconds after the wave front passing, increasing with time (see fig. 6, 7). This turned out to be completely unexpected. Any manipulations with the power and/or modulation of the radio signal do not affect the time dependence of anisotropy (see fig. 7). Thus, a peculiar effect of "memory" is registered, which requires a consistent explanation.

6. The passage of the reverse front of the acoustic wave changes the sign of the anisotropy arisen (see fig. 7).

\section{Discussion}

We attempted to describe quantitatively the effect observed considering the difference in the hydrodynamic behavior of the ions present in the solution. We have applied the simplest onedimensional pair model taking a cation and anion located at some distance from each other being immersed in a structureless medium having dielectric constant equal to 78.54. The solvent flow acceleration is exponentially rising with time in this model. There are two forces applied to each ion: the Coulomb force of attraction to another ion and the hydrodynamic Stokes drag because of the moving solvent that carries the ion along with it.

The hydration number for sodium cation was taken to be 16.6 [8], and 1.5 [9] for chloride-ion. The mass of the hydrated 
ion was estimated based on these numbers. The dielectric friction coefficients of $\mathrm{Na}^{+}$and $\mathrm{Cl}^{-}$were taken from [10]. We have evaluated the acceleration of the solvent flow, at which an equilibrium is reached between the Coulomb pulling force and the hydrodynamic forces that move the ions apart. The initial conditions for solving the system of differential equations were selected so that the distance between the ions $\mathrm{Na}+$ and $\mathrm{Cl}^{-}$approximately corresponded to the average in a $0.9 \%$ solution of $\mathrm{NaCl}(\approx 4.7 \mathrm{~nm})$ at the moment of equilibrium. As a result, for non-hydrated ions, according to our calculations, the equilibrium would occur upon acceleration of the solvent $\approx 8.4 \cdot 10^{12} \mathrm{~m} / \mathrm{s}^{2}$. For hydrated ions, the necessary acceleration would be $\approx 7.7 \cdot 10^{11} \mathrm{~m} / \mathrm{s}^{2}$.

First, the huge acceleration values obtained indicate the inconsistency of the simple continuum hydrodynamic model of two particles, in which there is no potential of mean force. An intensity of response from $\mathrm{KCl}$ solution also indicates the hydrodynamic explanation is at least insufficient. If our proposed hydrodynamic explanation were close to reality, then (ceteris paribus) it should have been much less than from $\mathrm{NaCl}$ solution because the dielectric friction coefficients for $\mathrm{K}^{+}$and $\mathrm{Cl}^{-}$ in water are very close to each other at the temperatures from $+5^{\circ} \mathrm{C}$ up to $+25^{\circ} \mathrm{C}$ [11, 12]. For the further development of the hydrodynamic model, it may be fruitful to use some new theoretical approaches that can describe the unsteady fluid flow at the microscopic level, for example, the so-called theories of complex fluids [13].

Secondly, it is very likely that a simple hydrodynamic description of the observed effect, even within the framework of a more perfect model, cannot correctly explain the phenomenon of residual anisotropy, the so-called "memory". Normally it should not have occurred and a oscillogram was predicted to show an ordinary curve of a differentiating chain when the oscilloscope input open. Taking into account the thermal motion in the solution, one should hardly assume the presence of any molecular vibrational processes that cause such a long "memory" - they undoubtedly should have been leveled out by thermal noise rather quickly. Moreover, the total energy of the thermal motion of water molecules is immeasurably greater than the energy introduced into the system by an acoustic pulse. The energy of a pulse with a duration of $50 \mu$ s emitted by a hydrophone with a power of $1 \mathrm{~W}$ was estimated to be of the order of $50 \mu \mathrm{J}$. The front of such a wave, according to our data, rotates the polarization scattering vector by about $10^{\circ}$. If we assume that the anisotropy is caused precisely by the orientation of the dipole moments of water molecules, then the integral hodograph of their thermal motion, which is a sphere in an isotropic medium, should become an ellipsoid with any significant difference of the major and minor semiaxes. It is difficult to hope that the acoustic pulse of the hydrophone used is capable of introducing sufficient energy for this, taking into account that the enthalpy of water at $+20^{\circ} \mathrm{C}$ is $84 \mathrm{~kJ} / \mathrm{kg}$ (relative to the triple point), and the mass of the solution in our experiment was about $2 \mathrm{~kg}$. The estimates of the energies of the acoustic pulse and the thermal motion of water differ by approximately 9 orders of magnitude.

Thus, in our opinion, the most likely physical process that remains suitable for explaining the observed giant relaxation times is, for example, the interaction of the nuclear spin angular momentums of hydrogen atoms of water molecules [14]. And the closest to the response we observe is, in our opinion, the process of magnetization of spin glass which is shown at figure $8[15]$.

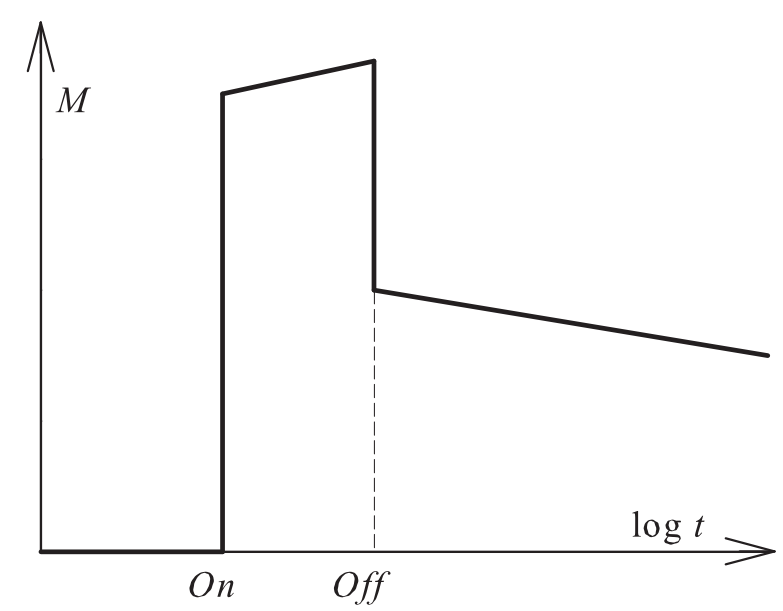

Figure 8: Magnetization $M$ of spin glass as a function of time after turning on and off the magnetic field

Using the properties of spin glass as a prototype, it can be assumed that the behavior of the solution studied in our experiments can be described by the Edwards-Anderson model [16]. That is, in the medium in which an acoustic wave propagates, there is not a random direction of spins, but degeneracy in various spin configurations, and the number of possible metastable states is infinitely large with a very long relaxation time. The authors cannot yet unambiguously correlate these states with the existing physical objects in the electrolyte solution (hydrated ions, individual water molecules, or their clusters) to unravel the problem. Of course, an exhaustive theoretical justification is required here. Also, some hope is given by the known experimental fact that two spin isomers of water can be separated under very specific conditions [17]. In this case the time that equilibrium between water isomers takes to establish, coincides (in order of magnitude) with the relaxation times we observe.

\section{Conclusions}

1. The rotation of the radio frequency signal polarization direction is highly likely caused by spin effects arising under the influence of an external force.

2. In our opinion, the effect will be widely used in biophysics and medicine, taking into account the need for non-contact research methods. Particularly promising is the monitoring of the state of the operator (car driver), where the most important is the heart rate and the integral assessment of the pumping function of the heart.

3. The practical application of the detected effect is already possible using the developed equipment and signal processing algorithms, since the proposed sensor will per- 
form the functions of a "finite state machine" (i.e. timeinvariant transcoding). The reference data in read-only memory is enough for it to work.

4. In addition to medical applications, the completely noncontact method, according to the authors, can be promising for industrial applications, for example, for monitoring liquids moving through pipelines, detecting unacceptable vibrations, turbulences, etc.

5. Especially important may be the use of a polarimetric sensor as a hydrophone for sea water. In this case, the phase transition on the path of sound waves from the medium in which they propagate to the sensitive element, as is the case in all existing microphones, is eliminated. It is possible that the sensitivity and selectivity of sonar stations can be increased.

6. Measurement of the angle of radio-frequency polarization direction after the passing of single short acoustic pulse through the body tissues will allow us to develop a fundamentally new method of single-pulse ultrasound introscopy.

7. We believe that the theoretical substantiation of the observed phenomenon requires wide cooperation of specialists in various fields

\section{Acknowledgments}

Our gratitude to prof. Alexander G. Nerukh, the head of the Department of Higher Mathematics of Kharkov National University of Radio Electronics ,to Igor I. Melnik, the associate professor of the Department of Organic Chemistry and Biotechnology of the National Technical University "Kharkov Polytechnic Institute", to Andrey N. Nechay, the researcher at the Institute of Microstructure Physics of the Russian Academy of Sciences for a fruitful discussion results and conclusions of the work.

\section{References}

[1] A. V. Kramarenko, G. P. Kulemin, A. M. Savchuk, Gemodinamicheskij komponent jelektrokardiogrammy, Biofizika 41 (1996) 686-694. URL: http://www.tredex-company.com/ru/ gemodinami-eskij-komponent-elektrokardiogrammy

[2] A. V. Kramarenko, G. P. Kulemin, A. M. Savchuk, 1996, The hemodynamic component of an electrocardiogram, URL: https://www.ncbi. nlm.nih.gov/pubmed/8924470

[3] A. V. Kramarenko, G. P. Kulemin, A. M. Savchuk, 1996, Hemodynamic contribution to human ecg, URL: http://www.tredex-company. com/sites/default/files/images/1996.pdf

[4] V. N. Tatarinov, S. V. Tatarinov, L. P. Ligthart, Vvedenie v sovremennuju teoriju poljarizacii radiolokacionnyh signalov. Poljarizacija ploskih jelektromagnitnyh voln i ee preobrazovanija, volume 2 of Polarizacija radiovoln, Izdatelstvo Tomskogo universiteta, 2006. URL: https://www . twirpx.com/file/2298809

[5] V. M. Shipulin, S. L. Andreev, J. J. Vecherskij, E. P. Gordov, Ispol'zovanie lazerov v serdechno-sosudistoj hirurgii: ot jeksperimenta k praktike, STT, 2010.

[6] The video of contactless radar electrocardiography working, 2019. URL: https://drive.google.com/open?id= 1YW2il87f-ElwXTDdIpLriGv2ldOLKzUR

[7] J. Rumble (Ed.), CRC Handbook of Chemistry and Physics, 100th Edition, CRC Press, 2019.
[8] V. M. Ivanov, K. A. Semenenko, G. V. Prohorova, E. F. Simonov, Natrij, Analiticheskaja himija jelementov, Nauka, 1986. URL: http:// chemteq.ru/assets/files/books/analytical/0065.djvu

[9] P. R. Smirnov, Structural parameters of the nearest surrounding of halide ions in the aqueous electrolyte solutions, Russian Journal of General Chemistry 83 (2013) 1469-1481. URL: https://doi.org/10.1134/ S107036321308001X doi 10.1134/S107036321308001X

[10] S. Koneshan, R. M. Lynden-Bell, J. C. Rasaiah, Friction coefficients of ions in aqueous solution at $25^{\circ} \mathrm{c}$, Journal of the American Chemical Society 120 (1998) 12041-12050. URL: https://doi.org/10.1021/ja981997x doi 10.1021/ja981997x arXiv:https://doi.org/10.1021/ja981997x

[11] M. Ueno, A. Yoneda, N. Tsuchihashi, K. Shimizu, Solvent isotope effect on mobilities of potassium and chloride ions in water at high pressure. ii. a low temperature study, The Journal of Chemical Physics 86 (1987) 46784683. URL: https://doi.org/10.1063/1.452708 doi 10.1063/ 1.452708 arXiv:https://doi.org/10.1063/1.452708

[12] M. Ueno, N. Tsuchihashi, K. Shimizu, Solvent isotope effect on mobilities of potassium and chloride ions in water at high pressure. iii. a high temperature study, The Journal of Chemical Physics 92 (1990) 25482552. URL: https://doi.org/10.1063/1.457947 doi 10.1063/ 1.457947 arXiv:https://doi.org/10.1063/1.457947

[13] B. Eisenberg, Mass action in ionic solutions, Chemical Physics Letters 511 (2011) 1-6. URL: http://www.sciencedirect.com/science/ article/pii/S0009261411006269 doi $10.1016 /$ j.cplett. 2011. 05.037

[14] J. Casanueva, J. S. Fabiàn, E. Dièz, A. Esteban, Nmr spin-spin coupling constants in water molecule: equilibrium and rovibrational values, Journal of Molecular Structure 565-566 (2001) 449 - 454. URL: http://www.sciencedirect.com/ science/article/pii/S0022286000009042 doi https : //doi.org/10.1016/S0022-2860(00)00904-2

[15] V. Kincel', Spinovye stekla kak model'nye sistemy dlja nejronnyh setej, Usp. fiz. nauk 152 (1987) 123-131. URL: https://ufn.ru/ru/ articles/1987/5/d/ doi 10.3367/UFNr.0152.198705d.0123

[16] S. F. Edwards, P. W. Anderson, Theory of spin glasses, Journal of Physics F: Metal Physics 5 (1975) 965-974. URL: https: //doi.org/10.1088\%2F0305-4608\%2F5\%2F5\%2F017 doi $10.1088 /$ 0305-4608/5/5/017

[17] V. I. Tikhonov, A. A. Volkov, Separation of water into its ortho and para isomers, Science 296 (2002) 2363-2363. URL: https:// science.sciencemag.org/content/296/5577/2363.full.pdf doi $10.1126 /$ science.1069513 